Original Research

\title{
Assessment of Trametes versicolor, Isochrysis galbana, and Purple Phototrophic Bacteria for the Removal of Pharmaceutical Compounds in Hospital Wastewater
}

Ana Cruz del Álamo ${ }^{1}$, María Isabel Pariente ${ }^{1}$, Alejandra Sanchez-Bayo ${ }^{2}$, Daniel Puyol ${ }^{1}$, Rosalía Rodríguez ${ }^{2}$, Victoria Morales ${ }^{1}$, Luis Fernando Bautista ${ }^{1}$, Gemma Vicente ${ }^{2}$, Juan Antonio Melero ${ }^{1}$, Raúl Molina ${ }^{1}$, Fernando Martínez Castillejo ${ }^{1, *}$

1. Department of Chemical and Environmental Technology. ESCET, Universidad Rey Juan Carlos, 28933, Móstoles, Madrid, Spain; E-Mails: ana.cruz.delalamo@urjc.es; isabel.pariente@urjc.es; daniel.puyol@urjc.es; $\quad$ victoria.morales@urjc.es; juan.melero@urjc.es; raul.molina@uric.es; fernando.castillejo@urjc.es

2. Department of Chemical, Energy and Mechanical Technology. ESCET, Universidad Rey Juan Carlos, 28933, Móstoles, Madrid, Spain; E-Mails: alejandra.sanchezbayo@urjc.es; rosalia.rodriguez@uric.es; gemma.vicente@urjc.es

*Correspondence: Fernando Martínez Castillejo; E-mail: fernando.castillejo@urjc.es

Academic Editor: Zed Rengel

Special Issue: Wastewater Treatment

Adv Environ Eng Res

2021, volume 2 , issue 4

doi:10.21926/aeer.2104027
Received: July 31, 2021

Accepted: October 12, 2021

Published: October 28, 2021

\begin{abstract}
Hospitals are one of the key contributors of pharmaceutical contaminants of emerging concern to the sewer systems. Hospitals wastewaters contain concentrations of pharmaceutical compounds between 3 and 150, which are higher than urban wastewater streams. However, dedicated treatments of the hospital effluents before discharge to the sewer system are not compulsory. Besides, conventional wastewater treatment plants have not been designed to remove pharmaceutical compounds effectively, and consequently, these micropollutants can reach the aquatic ecosystems. The removal of pharmaceutical compounds in real hospital wastewater was gaged using three different microbial cultures
\end{abstract}

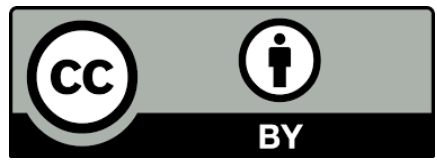

(C) 2021 by the author. This is an open access article distributed under the conditions of the Creative Commons by Attribution License, which permits unrestricted use, distribution, and reproduction in any medium or format, provided the original work is correctly cited. 
(white rot-fungus Trametes versicolor, microalga Isochrysis galbana, and a mixed culture of non-sulfur purple phototrophic bacteria). Before and after bioassays of the hospital wastewater, environmental hazard quotients were used to evaluate the biological treatment efficiency. Up to 45 out of the 79 compounds included in the analytical method were noticed in the hospital wastewater, with a predominance of analgesics/anti-inflammatories (acetaminophen, ibuprofen, ketoprofen, and naproxen). It was followed by antibiotics (azithromycin, ciprofloxacin, and ofloxacin, out of which the first two are included in the watch list of substances for monitoring in water in 2020) and anti-hypertensive drugs. Isochrysis galbana reached a reduction of $45 \%$ of the total concentration of pharmaceuticals, whereas Trametes versicolor and mixed culture of purple phototrophic bacteria improved the reductions up to $69 \%$ and $76 \%$, respectively. Moreover, potential environmental risk compounds (antibiotics, particularly ciprofloxacin and ofloxacin) were removed by Trametes versicolor in higher extension, obtaining a total hazard quotient reduction higher than the other two cultures. Removal efficiency and environmental risk assessment of remaining PhACs were used to evaluate the performance of the new biological systems for the treatment of emerging pollutants. According to both criteria, T. versicolor seems the most capable alternative for removing pharmaceutical compounds in hospital wastewater effluents.

\section{Keywords}

Pharmaceutical compounds; hospital wastewater; purple phototrophic bacteria; Trametes versicolor; Isochrysis galbana; environmental risk assessment

\section{Introduction}

Pharmaceutical active compounds (PhACs) are contaminants of emerging concern, progressively polluting the environment. These chemicals have biological activity, and they can be potentially hazardous contaminants due to their persistence and ubiquity, provoking undesired effects in the aquatic environment [1]. Frequently identified PhACs include various substances such as hormones, active principles and their metabolites, neuroactive drugs, antibiotics, or iodinated contrast media, among other drugs. Currently, no legal requirements have been established for discharging these biologically active substances into the surface water bodies [2-4]. Though some PhACs were identified for the first time as contaminants of emerging concern in 2013 and they were included in the list of priority substances in the Directive 2013/39/EU [5]. Later, ten substances or groups of pharmaceuticals were included in the Commission Implementing Decision (EU) 2015/495, as part of a watch list of substances for Union-wide monitoring in the field of water policy [6]. This watch list comprised diclofenac, 17- $\beta$-estradiol, estrone, 17- $\alpha$-ethynyl-estradiol, and macrolide antibiotics (erythromycin, clarithromycin, azithromycin). In 2018, the new Commission Implementing the Decision (EU) 2018/840 altered the substances, excluding diclofenac and including other antibiotics, such as amoxicillin and ciprofloxacin fluoroquinolone antibiotic [7]. The new Commission Implementing Decision (EU) 2020/1161 has newly retained amoxicillin and ciprofloxacin and replaced the other ones with sulfamethoxazole, trimethoprim, venlafaxine, and azoic compounds [8]. 
PhACs are detected in high sewage concentrations due to excretion and improper disposal of outdated or unused medication into the sewer system [9]. The Hospital and Medical Centre effluents show 150 times higher concentrations than urban wastewater [10-12]. Hence, they are high contributors of PhACs in the sewer systems. Dedicated treatment of hospital effluents before discharge to the sewer system is not compulsory [13]. The decentralized treatment would lessen the occurrence of PhACs in the sewer system, in centralized wastewater treatment plants (WWTP), and subsequently in the environment $[3,11]$.

Several technologies and methods are proposed and used to decrease PhACs in the water cycle [14]. Physical processes such as adsorption efficiently remove PhACs, but they are non-destructive techniques that merely cause a phase transfer of contaminants $[15,16]$. Advanced Oxidation Processes (AOPs), particularly ozonation and photochemical oxidation systems, are considered an interesting alternative for removing PhACs [17]. However, concerns related to the considerable energy and chemical consumption necessities are considered key obstacles to the commercial application of these processes [18]. Hence, the focus shifted from physical/chemical to novel biological systems to remove these pollutants.

Fungi exhibit a variety of strategies to remove toxic compounds, such as PhACs. Among them, the most common in literature are based on biotransformation or biodegradation mediated by different enzymatic systems and on the non-enzymatic process as bio-adsorption and bioprecipitation [19]. Additionally, the other strategy, less reported in the literature, is the so-called advanced bio-oxidation process, which exhibited the ability of Trametes versicolor, a white-rot fungus (WRF), to drive Fenton-like reactions and produce highly oxidizing $\mathrm{HO}^{\bullet}$ radicals through extracellular quinone redox cycling mechanisms [20-22]. Combining all these mechanisms provides an integral view of the versatility of applying WRF to eliminate a broad spectrum of xenobiotics, including PhACs [23, 24].

Various studies have established the efficacy of microalgae to remove PhACs, including analgesic (ibuprofen, naproxen, paracetamol, and ketoprofen)and antibiotics (ciprofloxacin, metoprolol, erythromycin, azithromycin, and amoxicillin) $[25,26]$. Besides, microalgae show remarkably higher tolerance to antibiotics than bacterial species, as they are not targeted for these active chemicals [27]. Nevertheless, microalgae still exhibited a low efficiency for others, such as carbamazepine. Particularly, Isochrysis galbana has been assessed as a substitute for the biodegradation of organic pollutants in natural environments due to its high antibiotic resistance, even in complex mixtures. Thus, the capacity of Isochrysis galbana to grow and partially bio-transform synthetic combinations of PhACs (antibiotics and antidepressant) has been demonstrated in the literature [28], being able to survive in the presence of antibiotics when mixed cultures of microalgae and bacteria were exposed to those compounds [29]. Moreover, these microalgae are defined by their robustness in a wide range of environmental conditions, such as salinity, temperature, other contaminants, and nutrient deficiencies [30-32].

The purple phototrophic bacteria (PPB) metabolism is highly complex. Several specific photoanaerobic metabolic pathways can degrade aromatic compounds like toluene, benzene [33], and heterocyclic aromatics [34]. Therefore, PPB can potentially be used as good microorganisms to bioremediate complex organic wastewaters bearing emerging contaminants, although no studies have been found for treating emerging contaminants so far [35]. Additionally, PPB has lower energetic requirements than traditional aerobic heterotrophs. PPB does not need oxygen and uses infrared sunlight as the energy source to assimilate organics and nutrients from the wastewater [36]. 
This work assesses three different biological cultures (white-rot fungus Trametes versicolor, microalga Isochrysis galbana, and a mixed culture of purple phototrophic bacteria) for the removal of PhACs in hospital wastewater. Besides, according to the European guidelines on risk assessment for new notified substances [37], the reduction of the environmental risk after each treatment was also estimated by calculating hazard quotient (HQ) indexes of each PhAC.

\section{Materials and Methods}

\subsection{Hospital Wastewater Samples}

Wastewater samples were taken from a hospital in the south of Madrid (Spain) during a weekly monitoring program using a typical sampling protocol described elsewhere $[38,39]$. The hospital generates three effluents coming from different therapeutic units. Three automatic water sampler systems were set up in each waste streamline to collect representative samples of the hospital's daily discharge. This system allowed taking $330 \mathrm{~mL}$ of wastewater every $10 \mathrm{~min}$ (110 mL of each streamline) for $24 \mathrm{~h}$, and after that, all the samples were mixed to obtain a homogeneous volume of ca. $48 \mathrm{~L}$. The samples were stored in polyethylene terephthalate (PET) reservoirs and preserved at four ${ }^{\circ} \mathrm{C}$ for 24 - $48 \mathrm{~h}$ until further characterization and preparation of the biological removal experiments. Table 1 shows the macroscopic definition of hospital wastewater.

Table 1 Main average characteristics of hospital wastewater samples (triplicate).

\begin{tabular}{|c|c|}
\hline Parameter & Average \\
\hline pH & $8.70 \pm 0.04$ \\
\hline Soluble Chemical Oxygen Demand ( $\left.\mathrm{COD}_{\text {soluble, }} \mathrm{mg} \mathrm{L}^{-1}\right)$ & $651 \pm 88$ \\
\hline Total solids (TS, $\mathrm{mg} \mathrm{L}^{-1}$ ) & $852 \pm 42$ \\
\hline Volatile solids (VS, $\mathrm{mg} \mathrm{L}^{-1}$ ) & $590 \pm 87$ \\
\hline Phosphorous as Phosphate $\left(\mathrm{P}_{-} \mathrm{PO}_{4}{ }^{3-}, \mathrm{mg} \mathrm{L}^{-1}\right)$ & $5.8 \pm 0.3$ \\
\hline Total Kjeldahl Nitrogen (TKN, $\mathrm{mg} \mathrm{L}^{-1}$ ) & $79 \pm 4$ \\
\hline Nitrogen as ammonium $\left(\mathrm{N}-\mathrm{NH}^{4+}, \mathrm{mg} \mathrm{L}^{-1}\right)$ & $50 \pm 5$ \\
\hline
\end{tabular}

\subsection{Cultures Preparation}

\subsubsection{White-Rot Fungus Trametes versicolor}

Trametes versicolor ( $T$. versicolor CECT 20817) was bought from the Colección Española de Cultivos Tipo (CECT). This culture was maintained by sub-culturing on $2 \%$ malt extract agar plates at $\mathrm{pH} 4.5$ and room temperature (ca. $25^{\circ} \mathrm{C}$ ) every 30 days [22]. Further, a mycelial suspension and pellets of $T$. versicolor were prepared following the procedure described by [40]. Briefly, the mycelial suspension was obtained by inoculating four pieces of $1 \mathrm{~cm}^{2}$ area of $T$. versicolor/agar plugs to a 500 $\mathrm{mL}$ Erlenmeyer flask containing $150 \mathrm{~mL}$ of malt extract medium (MEM) (20 g L ${ }^{-1}$ malt extract) adjusted to a $\mathrm{pH}$ of 4.5. The Erlenmeyer flasks were incubated on an orbital shaker at $80 \mathrm{rpm}$ and room temperature. - The MEM was removed by filtration after 7-9 days, and the dense mycelial mass was mixed with a homogenizer to obtain a mixed mycelial inoculum. Pellets were prepared by inoculating the fine mycelial inoculum into $250 \mathrm{~mL}$ of MEM in a $1 \mathrm{~L}$ Erlenmeyer flask, shaken for 5- 
6 days at the same conditions as above. The subsequent pellets were then washed with a Phosphate-Buffered Saline (PBS) solution (8 g L $\mathrm{LaCl}^{-1} 0.2 \mathrm{~g} \mathrm{~L}^{-1} \mathrm{KCl}, 1.42 \mathrm{~g} \mathrm{~L}^{-1} \mathrm{Na}_{2} \mathrm{HPO}_{4}, 0.24 \mathrm{~g} \mathrm{~L}^{-1}$ $\mathrm{KH}_{2} \mathrm{PO}_{4}$ at $\mathrm{pH} 4.5$ ) and blended together with a homogenizer to obtain fine particles that were finally used for the wastewater treatment experiments.

\subsubsection{Microalga Isochrysis galbana}

The Isochysis galbana (I. galbana, SAG 13.29) microalga strain was found at the University of Gottingen, Germany. The microalga was injected and cultivated in artificial seawater $\left(33.3 \mathrm{~g} \mathrm{~L}^{-1}\right.$ of Coral Marine Easy Mix, GroTech GmbH, Germany) and enriched using Walne medium (Aqualgae SL, Spain) for 14 days in a photo-bioreactor. The growth was obtained in a 5:45 volume ratio of microalga: culture medium, in alkaline conditions $(\mathrm{pH} 9-10)$ and at room temperature $\left(18-20^{\circ} \mathrm{C}\right)$. At 12:12 $\mathrm{h}$ light, darkness photoperiod was provided by artificial illumination with $12 \mathrm{~V}$ white LED lamps under a photon flux of $108 \mu \mathrm{mol} \cdot$ photon $\cdot \mathrm{m}^{-2} \cdot \mathrm{s}^{-1}\left(23.6 \mathrm{Wm} \mathrm{W}^{-2}\right.$ for radiation from $\left.400-700 \mathrm{~nm}\right)$. During the light periods, a $3.5 \mathrm{~L} \mathrm{~h}^{-1}$ of $\mathrm{CO}_{2}$ flow was supplied.

\subsubsection{Purple Phototrophic Bacteria (PPB)}

Mixed PPB culture was improved from domestic wastewater obtained from the pilot-scale wastewater treatment plant (WWTP) located at Universidad Rey Juan Carlos at Móstoles, Madrid (Spain), in a 10 ISO L bottle. The wastewater was supplemented with two gCoD L-1 of a mixture of acetate, propionate, butyrate, ethanol, and yeast extract with a COD ratio of 1:1:1:1:0.1. Ammonium chloride and sodium dihydrogen phosphate were then added to a COD: N:P ratio of 100:7.5:1.5. The culture was cultivated for seven days in anaerobic conditions at a $\mathrm{pH}$ value of 7.5, the stirring velocity was $120 \mathrm{rpm}$, and the temperature was $33^{\circ} \mathrm{C}$. The reactor was well-lit by $150 \mathrm{~W}$ infrared lamps and covered with specific Vis/UV absorbing foil [41], giving an average irradiance of $28 \mathrm{Wm}^{-2}$.

\subsection{Biological Removal Experiments}

\subsubsection{White-Rot Fungus Trametes versicolor}

Biological fungus experiments were done batch-wise in a $1 \mathrm{ISO}$ L Erlenmeyer flask using $500 \mathrm{~mL}$ of non-sterile raw hospital wastewater adjusted to a final $\mathrm{pH}$ of $4.5\left(2 \mathrm{~N} \mathrm{H}_{2} \mathrm{SO}_{4}\right)$. The bottles were injected with $100 \mathrm{mgvss} \mathrm{L}^{-1}$ of fungal pellets prepared as described previously. Similarly, $0.145 \mathrm{~g} \mathrm{~L}^{-1}$ $\mathrm{Fe}^{3+}$-oxalate hexahydrate, $0.085 \mathrm{~g} \cdot \mathrm{L}^{-1}$ gallic acid, and $0.0197 \mathrm{~g} \cdot \mathrm{L}^{-1} \mathrm{Mn}^{2+}$-nitrate monohydrate were added as advanced bio-oxidation promoters [20-22]. Lastly, flasks were incubated for five days at $300 \mathrm{rpm}$ and controlled temperature of $25^{\circ} \mathrm{C}$, as per the previous results obtained in literature [40].

\subsubsection{Microalga Isochrysis galbana}

The microalga was injected in a $500 \mathrm{ISO} \mathrm{mL}$ glass bottle with aeration for seven days, following previous studies evaluating the efficiency of high rate algal ponds in removing ciprofloxacin spiked at high concentrations in domestic wastewater [42]. The bottle was filled with a 5:45 vol. (100 mgvss $\mathrm{L}^{-1}$ ) microalga:hospital wastewater ratio and $50 \mathrm{mg} \mathrm{L}^{-1}$ of $\mathrm{NaNO}_{3}$ were added to provide the 
necessary nutrients for microalgae growth. The rest of the experimental conditions ( $\mathrm{pH}$, temperature, photoperiod, and $\mathrm{CO}_{2}$ flow) were similar to those used for culture preparation.

\subsubsection{Purple Phototrophic Bacteria (PPB)}

The experiments with a mixed culture of PPB were carried out in a $500 \mathrm{ISO} \mathrm{mL}$ glass bottle in anaerobic conditions inoculated with $100 \mathrm{~mL}$ of $100 \mathrm{mg} \mathrm{VSS} \mathrm{L}^{-1}$ of PPB mixed culture and then filled with hospital wastewater to a total volume of $500 \mathrm{~mL}$. Initial $\mathrm{pH}$ was fixed at 7.0 , the stirring velocity was $120 \mathrm{rpm}$, and the temperature was set at $30^{\circ} \mathrm{C}$. The orbital incubator shaker was illuminated for five days as described for this culture preparation. The reaction time was designated as per the previous works reporting reactors with hydraulic retention times of 2-10 days for the degradation of organic pollutants using mixtures of different PPB microbes, $R$. blasticus, $R$. adriaticus, Rhodo pseudomonas capsulatus, R. palustris, and Rhodovulum strictrum [43].

\subsection{Analytical Methods}

Chemical oxygen demand (COD) analysis was carried out by digestion of the sample with a commercially available solution containing potassium dichromate, sulfuric acid, and mercuric sulfate (II) (Test Spectroquant, Germany), following standard procedures [44]. The concentration of ammonia nitrogen was further examined following a photometric method with a commercial solution test (Test Spectroquant, Germany) at $690 \mathrm{~nm}$ using a UV/Vis spectrophotometer, analogous to the standard phenate method APHA 4500- $\mathrm{NH}_{3}$, in which indophenol (blue color) is obtained by the reaction of ammonia, hypochlorite, and phenol catalyzed by sodium nitroprusside [44]. Total Kjeldahl nitrogen (TKN) was recorded according to the titrimetric $4500-\mathrm{N}_{\text {org }} B$ method, in which nitrogen contained in organic compounds is transformed in ammonium nitrogen by reaction with $\mathrm{H}_{2} \mathrm{SO}_{4}$, potassium sulfate $\left(\mathrm{K}_{2} \mathrm{SO}_{4}\right)$, and cupric sulfate $\left(\mathrm{CuSO}_{4}\right)$ as the catalyst. Then it is titrated together with the existing ammonium nitrogen of the sample using a standard mineral acid [44]. P$\mathrm{PO}_{4}{ }^{3-}$ and total phosphorous were measured using the colorimetric method (Phosphate Cell Test Spectroquant, Germany) analogous to the common ascorbic acid method APHA 4500-PE. The $\mathrm{pH}$ was measured using a $\mathrm{pH} /$ ion-meter (Metrohm781, Herisau/Switzerland). Total solids (TS) and volatile solids (VS) were then analyzed according to standard methods [44] by drying at $105^{\circ} \mathrm{C}$ for $24 \mathrm{~h}$ to determine the TS and calcination in a furnace at $550{ }^{\circ} \mathrm{C}$ for $2 \mathrm{~h}$ to determine VS.

\subsection{Pharmaceuticals Analysis}

The analysis of pharmaceuticals was performed by ultra-high-performance liquid chromatography-tandem mass spectrometry (UHPLC-ESI-MS/MS) after solid-phase extraction (SPE) of the PhACs in the samples [45]. The analytical method includes 79 PhACs of 19 various therapeutic groups (Table S1). The accuracy of the SPE method was assessed according to the recovery values obtained after spiking the samples with a standard mixture of the PhACs at $50 \mathrm{ngL}^{-1}$ in triplicate [46]. Quantification was carried out based on the internal standard approach by the addition of the corresponding deuterated standards to all the samples and aqueous standards for preparing the calibration curve at a concentration of $0.01 \mathrm{ngL}^{-1}$ [45]. For quality assurance, three replicates per sample were examined to determine the analytical variability. Limits of detection (LODs) and quantification (LOQs) ranged from 0.1 to $25 \mathrm{ngL}^{-1}$ and 0.2 to $85 \mathrm{ngL}^{-1}$, respectively (Table S2). 


\subsection{Environmental Risk Assessment}

The environmental risk of water samples was projected using the hazard quotient (HQ) method. HQs were calculated for each target compound according to European guidelines [37] as shown in equation 1 [47]:

$$
H Q=\frac{M E C}{P N E C}
$$

Where MEC is the measured environmental concentration and PNEC accounts for the predicted non-effect concentration for the PhACs. In this research, the measured concentration of the PhACs in the different samples (raw wastewater and after treatment) was used as MEC $[39,48]$. If the quantified concentration was below the limit of detection (LOD) or the limit of quantification (LOQ), half of the LOQ was considered as MEC [13]. PNEC was attained following equation 2. The calculated PNEC values can be seen in Table $\mathbf{S 3 .}$

$$
P N E C=\frac{E C_{50} / L C_{50}}{A F}
$$

The numerator accounts for the toxicity concentration $\left(E C_{50}\right.$ or $L C_{50}$ ) to the most sensitive microorganism among three trophic levels (Fish, Daphnia, and Algae). In contrast, AF is an Assessment Factor established by European Commission [37]. In this study, the toxicity concentration $\left(\mathrm{EC}_{50} / \mathrm{LC}_{50}\right)$ for each PhAC was attained from the literature review, where the U.S. EPA Ecological Structure-Activity Relationships (ECOSAR) software was used for calculation in case of non-available data $[13,47,49]$. The assessment factor (AF) depends on the available data's uncertainty. It is inversely proportional to toxicity data availability and quality, ranging from 1 to 1000 [37]. Yet, this study has carefully chosen the most restrictive value ( $A F=1000)$, which is usually employed with acute $\mathrm{LC}_{50}$ toxicity values [13]. Therefore, PNEC is a value 1000 times lower than the toxicity concentration found for the most sensitive microorganism among Fish, Daphnia, and Algae.

\section{Results and Discussion}

\subsection{Compositional Distribution of PhACs in the Hospital Wastewater}

Analysis of PhACs showed that 45 out of the 79 analyzed compounds were noticed in the hospital wastewater (Table S4). The distribution of the key therapeutic groups of the hospital wastewater is depicted in Figure 1a. Antibiotics, analgesics, and anti-inflammatories were the most abundant therapeutic groups with percentages ranging from 22 to $33 \%$ and concentrations between 20-30 $\mu \mathrm{gL}^{-1}$. Compounds of these families correspond to the main types of drugs used in hospitals $[12,13]$. Anti-hypertensives and diuretics also had relevant contributions ranging between 17 and 13\%, followed by $\beta$-blockers with ca $5 \%$. This distribution is quite like others reported in the literature for hospital wastewaters $[12,48,50,51]$. It must also be pointed out that some PhACs detected in the hospital wastewater are currently included in the watch list of substances in the field of water policy by the Commission Implementing Decision (EU) 2018/840 [7]. It is the case of antibiotics, azithromycin, and erythromycin, with concentrations of 3647 and $0.94 \mathrm{ngL}^{-1}$, respectively, as well as ciprofloxacin, included in 2018 and 2020' watch lists, with a concentration upper 4000 ngL $^{-1}$, and 
sulfamethoxazole, included by Commission Implementing Decision (EU) 2020/1161 [8], with a concentration of ca. $1900 \mathrm{ngL}^{-1}$. Diclofenac, an analgesic omitted from the current watch list but included initially in the Commission Implementing Decision (EU) 2015/495, [6], also appeared in a concentration of $311 \mathrm{ngL}^{-1}$.

Anti-inflammatories are one of the most commonly used therapeutic groups in Spain. However, their consumption has reduced between 2013 and 2016 from 43.1 to 37.9 DHD, calculated as the total defined diary dose (DDD) of drug for each day per 1000 inhabitants [52]. In contrast, analgesic compounds grew their consumption between 2013 and 2020 from 24.9 to 37.5 DHD. Acetaminophen ( $>4000 \mathrm{ngL}^{-1}$ ), ibuprofen ( $>4000 \mathrm{ngL}^{-1}$ ), ketoprofen ( $8742 \mathrm{ngL}^{-1}$ ), and naproxen (12723 $\mathrm{ngL}^{-1}$ ) were the PhACs of this group with the highest concentrations. These results agree with the predominant consumption of ibuprofen and naproxen as the main analgesic drugs in Spain [52].

Antibiotics are also extensively used in hospitals, being one of the groups with higher loads in hospital wastewaters [51]. This therapeutic group is particularly critical as it can promote antibioticresistant bacteria (ARB) propagation into public wastewaters, breeding with the negative consequence of curative effects of antibiotics against humans and animals pathogens [53]. In Spain, the consumption of antibiotics ranged from 22 DHD in 1992 to 19.7 DHD in 2009, with an extraordinary increase in fluoroquinolones [54]. In this way, ciprofloxacin and ofloxacin, fluoroquinolones with similar characteristics, were the foremost antibiotics detected in the hospital wastewater, with concentrations higher than $4000 \mathrm{ngL}^{-1}$, followed by azithromycin and metronidazole.

The consumption of anti-hypertensive medications, including diuretics and beta-blocking agents, amplified from 185 DHD to 301 DHD in OECD countries from 2000 to 2020 [55]. In Spain, the use of anti-hypertensive medications tripled from 1992 (80.8 DHD) to 2020 (301 DHD), mainly valsartan, hydrochlorothiazide, furosemide, and atenolol [56]. The concentration of these PhACs in the hospital wastewater was among the highest of individual compounds. Therefore, the antihypertensive valsartan, the diuretics furosemide, and hydrochlorothiazide were present at 13559, 8173 , and $3707 \mathrm{ngL}^{-1}$, respectively, whereas the $\beta$-blocker atenolol reached a concentration of 4744 $\mathrm{ngL}^{-1}$.

Lastly, although the consumption of histamine $\mathrm{H} 2$ receptor antagonist has halved from 2000 (10.4 DHD) to 2012 (4.9 DHD) in Spain (AEMPS 2014), it is extraordinary the presence of ranitidine, a PhAC of this group with a concentration of $4038 \mathrm{ngL}^{-1}$. 


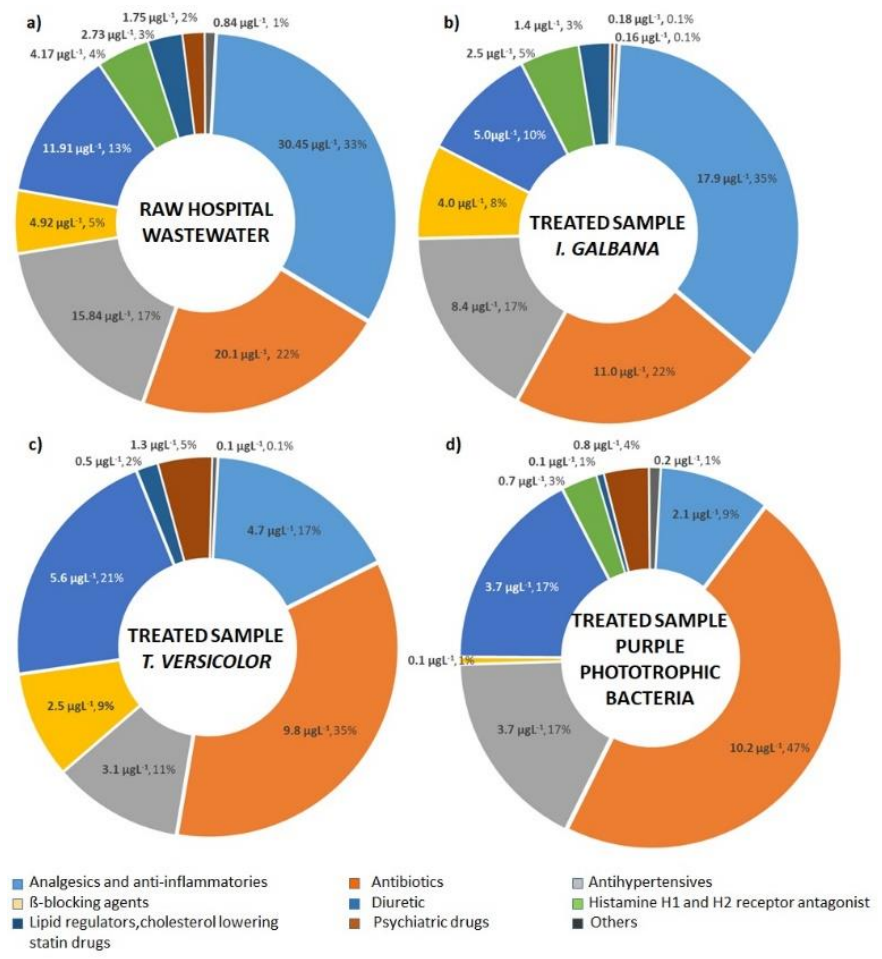

Figure 1 Distribution of the main therapeutic groups of PhACs for the (a) hospital wastewater and samples after experiments with the three tested microorganism's cultures (b), (c), and (d). Group of others includes drugs for prostatic hyperplasia, treat asthma, an anthelmintic, synthetic glucocorticoid, and calcium channel blockers.

\subsection{Performance of Biological Cultures for Removal of PhACs}

The distribution of the total amount of PhACs in the hospital wastewater samples after the biological treatment grouped by the main therapeutic families is displayed in figures $1 \mathrm{~b}, 1 \mathrm{c}$, and $1 \mathrm{~d}$. A remarkable removal of PhACs of the hospital wastewater was obtained for the experiments done with Trametes versicolor as fungal microorganism and mixed culture of purple phototrophic bacteria, attaining total reductions of ca. 70 and $77 \%$ of the initial concentration of PhACs. These results are owing to the high elimination of compounds belonging to the most abundant therapeutic groups such as analgesics/anti-inflammatories, antibiotics, and anti-hypertensives (84\%, 51\%, and 80\% for Trametes versicolor and 93\%, 49\%, and 76\% for purple phototrophic bacteria, respectively).

The fungus $T$. versicolor attained a remarkable removal of analgesics and anti-inflammatories in agreement with the results of previous studies [23]. Simultaneously, this culture achieved a COD removal of $35 \%$ and a fungus growth from 0.1 to $0.56 \mathrm{gL}^{-1}$ of VS. The removal of total PhACs is higher than that found in other studies under similar conditions. Other authors have stated a significant decrease of $83 \%$ for the total concentration of pharmaceutical compounds in hospital wastewater but working under sterile conditions [50]. Still, the efficiency decreased to ca. 53\% when non-sterile conditions were used. In that study, 51 pharmaceuticals over 99 analyzed were detected in the initial hospital wastewater, and high elimination degrees were attained for analgesic/antiinflammatories and antibiotics (ca. 99 and 86\%, respectively) [50]. The potential capacity of $T$. versicolor for removing PhACs is associated with their ability to generate hydrogen peroxide by their unspecific extracellular oxidative enzymatic system that can promote highly oxidizing hydroxyl 
radicals through subsequent Fenton-like reactions. These oxidizing radicals can oxidize and break down the bonds of PhACs, which makes them vulnerable to enzymatic attack and bio-oxidation mechanisms of fungal transformation [19]. The production of hydroxyl radicals is improved by adding quinone-like mediators in the presence of chelated ferric ions. The same has been done in this work in the so-called advanced bio-oxidation process.

The non-adapted purple phototrophic bacteria culture seems to catabolize the organics present in the wastewater, as can be deduced by the COD consumption (36\%) and high PhACs removal but their growth (from 0.1 to $0.19 \mathrm{gL}^{-1}$ of VS) is limited in this study. The purple phototrophic bacteria metabolism is highly diverse and includes aromatics breakdown [34], as well degradation of toxic substituents from organic compounds like azo-dyes and halogenated substituents [57]. This suggests that an adaptation period could promote the mechanisms of bactericides degradation, giving rise to the improvement of biomass growth and specific activities, as previously reported for other toxic wastewater $[58,59]$. These results represent a promising eco-friendly alternative that should be explored in more detail in the future, considering the lack of works using this microorganism in the bioremediation of hospital effluents.

Regarding the I. galbana microalga, this culture showed a lower removal of the initial concentration of PhACs (ca. 45\%). The reduction of most large therapeutic groups, analgesics/antiinflammatories, antibiotics, and anti-hypertensive, were $41 \%, 45 \%$, and $47 \%$, respectively. In particular, naproxen and ibuprofen displayed a low reduction, predominant PhACs of the group of analgesics and anti-inflammatories in the raw hospital wastewater (see Table S4). These results agree with the low removal observed with a mixed microalgae culture for this therapeutic group in the treatment of toilet waters, achieving a naproxen removal of ca. 10\% [60]. Nevertheless, other microalgae cultures such as Chlorella vulgaris and Scenedesmus obliquus from lagoon water demonstrated higher removal efficiencies with ibuprofen removals of ca. 60\% [61] or almost complete removal using $C$. sorokiniana (99\%) from anaerobically treated black water and synthetic urine [62] or a mixed microalgae culture taken from a real lake (98.5\%) [60]. Here, the lower efficiency of the microalgae in terms of PhACs removal is in contrast with the microalgae growth (from 0.1 to $0.65 \mathrm{gL}^{-1}$ of VS) and COD removal (43\%) in this study. I. galbana can grow under mixotrophic conditions using organic matter as a carbon source [63]. Moreover, the literature has reported that antibiotics can partially inhibit the biological activity of marine microalgae [32]. Still, at the same time, some microalgae can activate a self-defense mechanism consisted of increasing their carotenoid content as the protective process for the photosynthetic system and concomitant algal growth and survival [25]. This mechanism seems to be predominant in the case of I. galbana, as this microalga proves a high resistance to PhACs compared to other marine microalgae [32].

It is also interesting to show that overall removal degrees of the antibiotic therapeutic group were alike for the three cultures (ca. 50\%). Still, the individual elimination of each antibiotic compound was significantly different depending on the culture. Thus, T. versicolor allowed a remarkable reduction of ciprofloxacin (50\%), a PhAC included in the watch list in 2018 and 2020, which was refractory to I. galbana and purple phototrophic bacteria. The high efficiency of $T$. versicolor for ciprofloxacin removal has already been reported in the literature, but operating with a longer incubation time (15 days) [47]. Azithromycin and ofloxacin, also included in the watch list, were almost eliminated with I. galbana ( $88 \%$ and $99 \%$, respectively), being less efficient for purple phototrophic bacteria (65\% and $6 \%$, respectively) and T. versicolor ( $22 \%$ and $63 \%$, respectively). The results of microalgae and purple phototrophic bacteria are under a preliminary comparative study 
between photo-bioreactors of both types of microorganisms, where non-sterile Chlorella vulgaris culture was more effective than bacteria in the removal of detected antibiotic drugs [64]. Concerning $T$. versicolor, previous studies have also reported a variable removal rate of ofloxacin ranging from 46 to $99 \%$, but working under favorable conditions for the culture such as sterile conditions, the addition of external nutrient sources (glucose and ammonium tartrate), continuous aeration and longer incubation times of 12 and 15 days $[47,65]$.

The removal of PhACs determined in this work could not distinguish between biodegradation and bio-absorption of the different microorganism cultures. However, in the case of Trametes versicolor, note that recent results have demonstrated that only between 3 and $13 \%$ of PhACs elimination can be attributed to sorption processes $[40,66]$.

\subsection{Environmental Risk Assessment of PhACs}

The hazard quotients ( $\mathrm{HQ}$ ) of individual and grouped PhACs detected in the hospital wastewater and samples after treatment with the three cultures are shown in Figure 2. HQs of all individual compounds are briefed in Table S5. The initial $\Sigma \mathrm{HQ}$ value of the raw hospital wastewater was 1884. The remaining PhACs for samples after the treatment with the different cultures were 1289, 1273, and 951 for I. galbana, purple phototrophic bacteria, and T. versicolor, respectively. As per these results, T. versicolor attained the highest environmental risk reduction (ca. 50\%), whereas microalgae and purple phototrophic bacteria accomplished a lower reduction of ca. $32 \%$ in both cases.
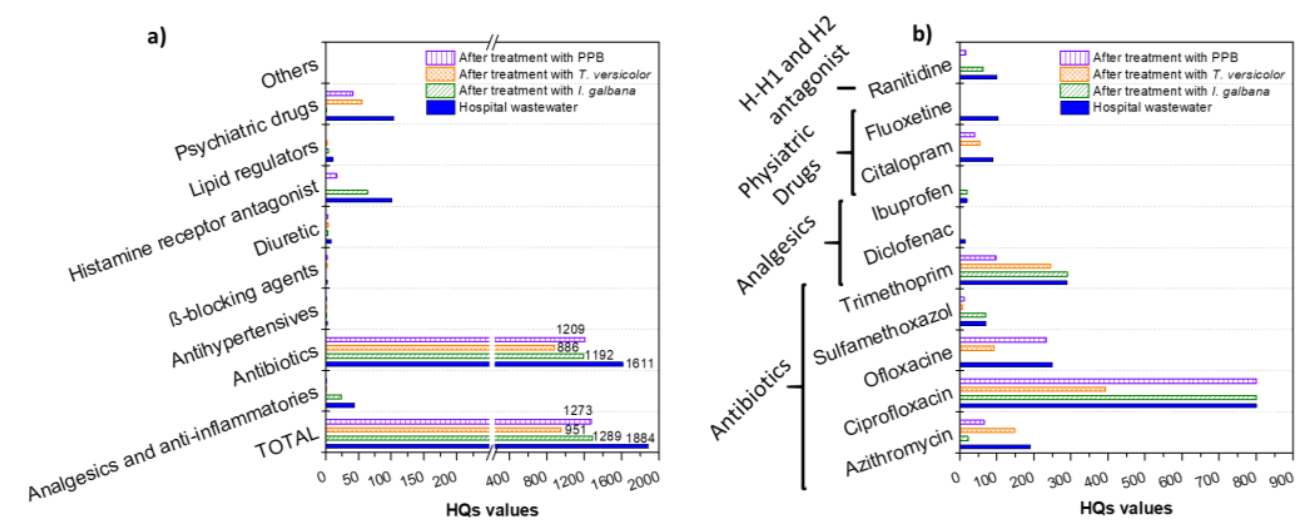

Figure 2 Contribution of therapeutic groups (a) and more hazardous PhACs (HQ>10) (b) to the total HQ of the hospital wastewater and the treated samples.

The comparison of HQs by therapeutic groups (Figure 2a) reveals a key contribution of antibiotics to the total $\mathrm{HQ}$ in all the samples. Thus, the role of antibiotics to the total $\mathrm{HQ}$ accounts for $85 \%$ of the hospital wastewater, a value of 1611 over the total HQ of 1884. Other studies examining entire hazardous quotients for real hospital wastewaters have already reported this high contribution of antibiotics. This therapeutic group represented between 85 and $92 \%$ of the total HQ [39, 67].

The PhACs can be classified into four groups as per their environmental risk using the criteria of Stockholm County Council [13], [68]: insignificant (HQ less or equal 0.1), low (HQ between 0.1 and 1), moderate (HQ between 1 and 10) and high (HQ greater than 10). Figure $\mathbf{2 b}$ shows the $10 \mathrm{PhACs}$ with high environmental risk according to their $\mathrm{HQ}>10$ in the hospital wastewater. All the 
antibiotics are PhACs with high ecological hazards due to their low PNEC values, such as ciprofloxacin, ofloxacin, azithromycin, and sulfamethoxazole with values as low as 0.005, 0.0016, 0.002 or $0.027 \mathrm{\mu L}^{-1}$, respectively (see Table S3). Among antibiotics, ciprofloxacin embodies a significant contribution to all wastewater samples, $42 \%$ in the hospital wastewater $(\mathrm{HQ}=800$ over a total HQ value of 1884). Its low reduction with I. galbana and purple phototropic bacteria makes its contribution in the treated samples quite relevant (ca. $62 \%$ in both samples and HQ values around 800). In the case of $T$. versicolor, the HQ reduced significantly due to the higher removal of ciprofloxacin. However, the contribution of all antibiotics to the total HQ was still significant (44\%). Regarding PhACs of other therapeutic groups, diclofenac and fluoxetine also have HQs $>10$ (about 16 and 14, respectively). However, their potential risk becomes low or insignificant after the treatments. In terms of citalopram, a psychiatric drug with an initial $\mathrm{HQ}$ value in the hospital wastewater of 90, only the treatment with I. galbana allows an almost complete reduction, achieving a final $\mathrm{HQ}$ of ca. 0.2. In the treated samples with $T$. versicolor and purple phototrophic bacteria, final values of 54 and 40, respectively, were estimated. Conversely, the environmental risk of ranitidine with an $\mathrm{HQ}$ of 100 in the raw hospital wastewater was only reduced after treatment with $T$. versicolor. At the same time, values of 63 and 16 remained after treatment with I. galbana and purple phototrophic bacteria.

\subsection{Overall Assessment of Biological Cultures for Treatment of the Hospital Wastewater}

The efficiency in removing PhACs and reducing the hazard quotient after the experiments of the three cultures is summarized in Figure 3. Interestingly, I. galbana and purple phototrophic bacteria displayed distinct elimination ratios of the overall PhACs ( $45 \%$ and $76 \%$, respectively) but similar $\mathrm{HQ}$ reduction (ca. 32\%) in both cases. This fact indicates that removing particular drugs of high environmental risk is quite important in evaluating the feasibility of the biological culture for potential future implementation. In this sense, T. versicolor achieved a major removal of PhACs and a higher reduction of the environmental risk, mainly due to the HQ reduction of ciprofloxacin (ca. $50 \%$ ). Nevertheless, this reduction was not as high as that reported in the literature for treating hospital wastewater using fungi (80-90\%). Still, it must be noted that the treatment incubation in that study was eight days [67] instead of the five days of this work.

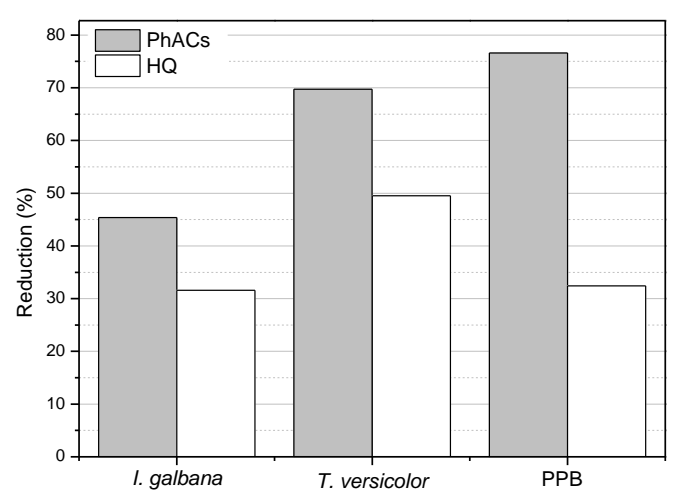

Figure 3 Comparison of the efficiency of the three cultures in terms of chemical elimination (PhACs) and environmental risk reduction (HQ). 
These results demonstrate that novel wastewater treatments technologies for the elimination of emerging pollutants like PhACs, cannot be only evaluated from a removal point of view for PhACs. A complementary analysis of indicators regarding the potential environmental risk of remaining compounds after tested treatments, such as the HQ index, is necessary because it depends not only on the overall pharmaceutical loading but also on the presence of hazardous compounds with high environmental risk.

\section{Conclusions}

The hospital wastewater contains various pharmaceutical residuals, some of them characterized by high risk to aquatic organisms $(\mathrm{HQ}>10)$, particularly the antibiotic ciprofloxacin $(\mathrm{HQ}=800)$. The three studied microbial cultures produce a substantial reduction of the PhACs content of the hospital wastewater. Remarkable PhACs removals were accomplished for $T$. versicolor and purple phototrophic bacteria cultures (70 and $77 \%$, respectively), whereas the microalgae culture showed a lower elimination (45\%). Moreover, $T$. versicolor allowed an $\mathrm{HQ}$ reduction of $\mathrm{ca} .50 \%$, as compared to values of ca. $32 \%$ of both I. galbana and purple phototrophic bacteria cultures. This fact is mainly due to the higher removal of $T$. versicolor for ciprofloxacin, one of the most hazardous compounds in the HQ. According to the removal of PhACs and the reduction of the hazard quotients of the remaining PhACs, the white-rot fungus, $T$. versicolor, seems to be the more promising biological culture for the treatment of hospital wastewater. However, the performance of a biological system based on white-rot fungi needs to be optimized to improve its efficiency for eliminating PhACs with low values of PNEC and high environmental risk potential.

\section{Acknowledgments}

Institut Català de Recerca de l'Aigua (ICRA) is also acknowledged for the pharmaceutical analysis performed in this work.

\section{Additional Materials}

The following additional materials are uploaded to the page of this paper.

1. Table S1: Target compounds organized by the therapeutical groups and the isotopically labelled internal standards assigned for quantification.

2. Table S2: Limit of detection (LOD) and limit of quantification (LOQ).

3. Table S3: Average concentration $(n=3)$ of the target pharmaceutical compounds $\left(n g \cdot L^{-1}\right)$ in the initial and final hospital wastewater samples with each culture.

4. Table S4: PNEC for the target pharmaceutical compounds $\left(\mu \mathrm{L} \mathrm{L}^{-1}\right)$.

5. Table S5: Hazard quotients (HQ) in the initial and final hospital wastewater samples with each culture.

\section{Author Contributions}

Ana Cruz del Álamo: Investigation, Writing- Original draft, Formal Analysis, Data Curation, Writing - Review \& Editing; María Isabel Pariente: Formal Analysis, Data Curation, Supervision, Validation, Writing - Review \& Editing; Alejandra Sanchez-Bayo: Investigation and Writing - Review 
\& Editing; Daniel Puyol: Writing - Review \& Editing; Rosalía Rodríguez: Writing - Review \& Editing; Victoria Morales: Writing - Review \& Editing; Luis Fernando Bautista: Writing - Review \& Editing, Funding acquisition; Gemma Vicente: Writing - Review \& Editing, Funding acquisition; Juan Antonio Melero: Writing - Review \& Editing, Funding acquisition; Fernando Martínez: Conceptualization, Supervision, Validation, Writing - Review \& Editing, Funding acquisition; Raúl Molina: Conceptualization, Writing- Original draft, Supervision, Validation, Writing - Review \& Editing.

\section{Funding}

Authors thank the financial support of the Regional Government of Madrid provided through project REMTAVARES S2018/EMT-4341 and INSPIRA1 (S2013/ABI-2783), both co-financed by the European Social Fund ("Una manera de hacer Europa").

\section{Competing Interests}

The authors declare that they have no known competing financial interests or personal relationships that could have appeared to influence the work reported in this paper.

\section{References}

1. Taheran M, Naghdi M, Brar SK, Verma M, Surampalli RY. Emerging contaminants: Here today, there tomorrow! Environ Nanotechnol Monit Manag. 2018; 10: 122-126.

2. Gogoi A, Mazumder P, Tyagi VK, Chaminda GG, Kyoungjin An A, Kumar M. Occurrence and fate of emerging contaminants in water environment: A review. Groundw Sustain Dev. 2018; 6: 169180.

3. Rodriguez-Narvaez OM, Peralta-Hernandez JM, Goonetilleke A, Bandala ER. Treatment technologies for emerging contaminants in water: A review. Chem Eng J. 2017; 323: 361-380.

4. Gomez-Olivan LM. Legislation controlling the discharge of pharmaceuticals into the environment. Ecopharmacovigilance: Multidisciplinary approaches to environmental safety of medicines. New York: Springer, Cham; 2018.

5. Directive 2013/39/EU of the European Parliament and of the Council amending Directives 2000/60/EC and 2008/105/EC as regards priority substances in the field of water policy [Internet]. European Union; 2013. Available from: https://www.ecolex.org/details/legislation/directive-201339eu-of-the-european-parliamentand-of-the-council-amending-directives-200060ec-and-2008105ec-as-regards-prioritysubstances-in-the-field-of-water-policy-lex-faoc127344/.

6. Commission Implementing Decision (EU) 2015/495 establishing a watch list of substances for Union-wide monitoring in the field of water policy pursuant to Directive 2008/105/EC of the European Parliament and of the Council [Internet]. European Union; 2015. Available from: https://www.ecolex.org/details/legislation/commission-implementing-decision-eu-2015495establishing-a-watch-list-of-substances-for-union-wide-monitoring-in-the-field-of-waterpolicy-pursuant-to-directive-2008105ec-of-the-european-parliament-and-of-the-council-lexfaoc142647/.

7. Commission Implementing Decision (EU) 2018/495 establishing a watch list of substances for Union-wide monitoring in the field of water policy pursuant to Directive 2008/105/EC of the 
European Parliament and of the Council [Internet]. European Union; 2015. Available from: https://eur-lex.europa.eu/legal-content/EN/TXT/?uri=CELEX\%3A32018D0840.

8. Commission Implementing Decision (EU) 2020/1161 of 4 August 2020 establishing a watch list of substances for Union-wide monitoring in the field of water policy pursuant to Directive 2008/105/EC of the European Parliament and of the Council (notified under document number C(2020) 5205) (Text with EEA relevance) [Internet]. European Union; 2020. Available from: https://eur-lex.europa.eu/legalcontent/EN/TXT/?uri=uriserv:OJ.L .2020.257.01.0032.01.ENG\&toc=OJ:L:2020:257:TOC.

9. Boix C, Ibáñez M, Fabregat-Safont D, Morales E, Pastor L, Sancho JV, et al. Behaviour of emerging contaminants in sewage sludge after anaerobic digestion. Chemosphere. 2016; 163: 296-304.

10. Lien LT, Hoa NQ, Chuc NT, Thoa NT, Phuc HD, Diwan V, et al. Antibiotics in wastewater of a rural and an Urban Hospital before and after wastewater treatment, and the relationship with antibiotic use - a one year study from Vietnam. Int J Environ Res Public Health. 2016; 13: 588.

11. Verlicchi P, Al Aukidy M, Galletti A, Petrovic M, Barceló D. Hospital effluent: Investigation of the concentrations and distribution of pharmaceuticals and environmental risk assessment. Sci Total Environ. 2012; 430: 109-118.

12. Verlicchi P, Galletti A, Petrovic M, Barceló D. Hospital effluents as a source of emerging pollutants: An overview of micropollutants and sustainable treatment options. J Hydrol. 2010; 389: 416-428.

13. Mendoza A, Aceña J, Pérez S, Lopez de Alda M, Barceló D, Gil A, et al. Pharmaceuticals and iodinated contrast media in a hospital wastewater: A case study to analyse their presence and characterise their environmental risk and hazard. Environ Res. 2015; 140: 225-241.

14. Phoon BL, Ong CC, Mohamed Saheed MS, Pau-Loke S, Chang JS, Chuan Ling T, et al. Conventional and emerging technologies for removal of antibiotics from wastewater. J Hazard Mater. 2020; 400: 122961.

15. Rizzo L, Malato S, Antakyali D, Beretsou VG, Dolic MB, Gernjak W, et al. Consolidated vs new advanced treatment methods for the removal of contaminants of emerging concern from urban wastewater. Sci Total Environ. 2019; 655: 986-1008.

16. Sophia AC, Lima EC. Removal of emerging contaminants from the environment by adsorption. Ecotoxicol Environ Saf. 2018; 150: 1-17.

17. Kanakaraju D, Glass BD, Oelgemöller M. Advanced oxidation process-mediated removal of pharmaceuticals from water: A review. J Environ Manag. 2018; 219: 189-207.

18. Souza FS, Da Silva VV, Rosin CK, Hainzenreder L, Arenzon A, Pizzolato T, et al. Determination of pharmaceutical compounds in hospital wastewater and their elimination by advanced oxidation processes. J Environ Sci Health A. 2018; 53: 213-221.

19. Olicón-Hernández DR, González-López J, Aranda E. Overview on the biochemical potential of filamentous fungi to degrade pharmaceutical compounds. Front Microbiol. 2017; 8: 1792.

20. Cruz del Álamo A, Pariente MI, Martínez F, Molina R. Trametes versicolor immobilized on rotating biological contactors as alternative biological treatment for the removal of emerging concern micropollutants. Water Res. 2020; 170: 115313.

21. Cruz del Álamo A, Pariente MI, Vasiliadou I, Padrino B, Puyol D, Molina R, et al. Removal of pharmaceutical compounds from urban wastewater by an advanced bio-oxidation process based on fungi Trametes versicolor immobilized in a continuous RBC system. Environ Sci Pollut 
Res. 2018; 25: 34884-34892.

22. Marco-Urrea E, Pérez-Trujillo M, Cruz-Morató C, Caminal G, Vicent T. Degradation of the drug sodium diclofenac by Trametes versicolor pellets and identification of some intermediates by NMR. J Hazard Mater. 2010; 176: 836-842.

23. Mir-Tutusaus JA, Baccar R, Caminal G, Sarrà M. Can white-rot fungi be a real wastewater treatment alternative for organic micropollutants removal? A review. Water Res. 2018; 138: 137-151.

24. Mir-Tutusaus JA, Masís-Mora M, Corcellas C, Eljarrat E, Barceló D, Sarrà M, et al. Degradation of selected agrochemicals by the white rot fungus Trametes versicolor. Sci Total Environ. 2014; 500-501: 235-242.

25. Xiong JQ, Kurade $\mathrm{MB}$, Jeon $\mathrm{BH}$. Can microalgae remove pharmaceutical contaminants from water? Trends Biotechnol. 2018; 36: 30-44.

26. Matamoros V, Gutiérrez R, Ferrer I, García J, Bayona JM. Capability of microalgae-based wastewater treatment systems to remove emerging organic contaminants: A pilot-scale study. J Hazard Mater. 2015; 288: 34-42.

27. Liu G, Zhou J, Wang J, Song Z, Qv Y. Bacterial decolorization of azo dyes by Rhodopseudomonas palustris. World J Microbiol Biotechnol. 2006; 22: 1069-1074.

28. Hom-Diaz A, Vicent T, Blanquez P. Degradation of pharmaceutical compounds by microalgae: Photobioreactor wastewater treatment, biomass harvesting and methanization. Barcelona, Spain: Universitat Autònoma de Barcelona; 2016.

29. Molina-Cárdenas CA, Sánchez-Saavedra M, Licea-Navarro A. Decreasing of bacterial content in Isochrysis galbana cultures by using some antibiotics. Rev Biol Mar Oceanogr. 2016; 51: 101112.

30. Ammar SH, Khadim HJ, Mohamed Al. Cultivation of Nannochloropsis oculata and Isochrysis galbana microalgae in produced water for bioremediation and biomass production. Environ Technol Innov. 2018; 10: 132-142.

31. Tato T, Salgueiro-González N, León VM, González S, Beiras R. Ecotoxicological evaluation of the risk posed by bisphenol $A$, triclosan, and 4-nonylphenol in coastal waters using early life stages of marine organisms (Isochrysis galbana, Mytilus galloprovincialis, Paracentrotus lividus, and Acartia clausi). Environ Pollut. 2018; 232: 173-182.

32. Teixeira JR, Granek EF. Effects of environmentally-relevant antibiotic mixtures on marine microalgal growth. Sci Total Environ. 2017; 580: 43-49.

33. Sasikala C, Ramana CV. Biodegradation and metabolism of unusual carbon compounds by anoxygenic phototrophic bacteria. Adv Microb Physiol. 1998; 39: 368-377.

34. Harwood CS. Degradation of aromatic compounds by purple nonsulfur bacteria. In: The purple phototrophic bacteria. Dordrecht: Springer Netherlands; 2009.

35. De las Heras I, Padrino B, Molina R, Segura Y, Melero JA, Mohedano AF, et al. Efficient treatment of synthetic wastewater contaminated with emerging pollutants by anaerobic purple phototrophic bacteria. In: Frontiers in wastewater treatment and modelling. New York: Springer, Cham; 2017.

36. Capson-Tojo G, Batstone DJ, Grassino M, Vlaeminck SE, Puyol D, Verstraete W, et al. Purple phototrophic bacteria for resource recovery: Challenges and opportunities. Biotechnol Adv. 2020; 43: 107567.

37. Comission. Technical Guidance Document on Risk Assessment in support of Commission 
Directive 98/67/EEC on Risk Assessment for new notified substances, Commission Regulation (EC) No 1488/94 on Risk Assessment for existing substances, and Directive 98/8/EC of the Euro 2003. Available from: https://op.europa.eu/en/publication-detail/-/publication/212940b83e55-43f8-8448-ba258d0374bb.

38. Cruz del Álamo A, González C, Pariente MI, Molina R, Martínez F. Fenton-like catalyst based on a reticulated porous perovskite material: Activity and stability for the on-site removal of pharmaceutical micropollutans in a hospital wastewater. Chem Eng J. 2020; 401: 126113.

39. Segura Y, Cruz del Álamo A, Munoz M, Álvarez-Torrellas S, García J, Casas JA, et al. A comparative study among catalytic wet air oxidation, Fenton, and Photo-Fenton technologies for the on-site treatment of hospital wastewater. J Environ Manag. 2021; 290: 112624.

40. Vasiliadou IA, Sánchez-Vázquez R, Molina R, Martinez F, Melero JA, Bautista LF, et al. Biological removal of pharmaceutical compounds using white-rot fungi with concomitant FAME production of the residual biomass. J Environ Manag. 2016; 180: 228-237.

41. Hülsen $T$, Batstone DJ, Keller J. Phototrophic bacteria for nutrient recovery from domestic wastewater. Water Res. 2014; 50: 18-26.

42. Hom-Diaz A, Norvill ZN, Blánquez P, Vicent T, Guieysse B. Ciprofloxacin removal during secondary domestic wastewater treatment in high rate algal ponds. Chemosphere. 2017; 180: 33-41.

43. Talaiekhozani A, Rezania S. Application of photosynthetic bacteria for removal of heavy metals, macro-pollutants and dye from wastewater: A review. J Water Process Eng. 2017; 19: 312-321.

44. APHA, AWWA, WEF. Standard methods for examination of water and wastewater. Washington, DC: American Public Health Association; 2012.

45. Gros M, Rodríguez-Mozaz S, Barceló D. Fast and comprehensive multi-residue analysis of a broad range of human and veterinary pharmaceuticals and some of their metabolites in surface and treated waters by ultra-high-performance liquid chromatography coupled to quadrupolelinear ion trap tandem. J Chromatogr A. 2012; 1248: 104-121.

46. Hernández-Ramírez A, Hernández-Tenorio R, Hinojosa-Reyes L, Ramos-Delgad N, HernándezRamírez A. Determination of pharmaceuticals discharged in wastewater from a public hospital using LC-MS/MS technique. J Mex Chem Soc. 2021; 65: 94-108.

47. Lucas D, Badia-Fabregat M, Vicent T, Caminal G, Rodriguez- Mozaz S, Balcázar JL, et al. Fungal treatment for the removal of antibiotics and antibiotic resistance genes in veterinary hospital wastewater. Chemosphere. 2016; 152: 301-308.

48. Santos LH, Gros M, Rodriguez-Mozaz S, Delerue-Matos C, Pena A, Barceló D, et al. Contribution of hospital effluents to the load of pharmaceuticals in urban wastewaters: Identification of ecologically relevant pharmaceuticals. Sci Total Environ. 2013; 461-462: 302-316.

49. Sanderson H, Johnson DJ, Wilson CJ, Brain RA, Solomon KR. Probabilistic hazard assessment of environmentally occurring pharmaceuticals toxicity to fish, daphnids and algae by ECOSAR screening. Toxicol Lett. 2003; 144: 383-395.

50. Cruz-Morató C, Lucas D, Llorca M, Rodríguez-Mozaz S, Gorga M, Mira P, et al. Hospital wastewater treatment by fungal bioreactor: Removal efficiency for pharmaceuticals and endocrine disruptor compounds. Sci Total Environ. 2014; 493: 365-376.

51. Oliveira TS, Al Aukidy M, Verlicchi P. Occurrence of common pollutants and pharmaceuticals in hospital effluents. In: Hospital wastewaters: Characteristics, management, treatment and environmental risks. New York: Springer, Cham; 2018. 
52. Utilización de medicamentos antiinflamatorios no esteroides (AINE) en España durante el periodo 2013-2016. Spain: AEMPS; 2017; U/AIN/V1/11/09/2017.

53. Inyinbor AA, Bello OS, Fadiji AE, Inyinbor HE. Threats from antibiotics: A serious environmental concern. J Environ Chem Eng. 2018; 6: 784-793.

54. Uso de antibioticos en España [Internet]. Spain: AEMPS; 2010. Available from: https://www.aemps.gob.es/medicamentosUsoHumano/observatorio/docs/antibioticos.pdf.

55. AEMPS. Utilización de medicamentos antihipertensivos en España. Informe 2021. Available from:

https://app.powerbi.com/view?r=eyJrljoiNWE2MTY4MGQtZWY4Zi00YTBhLTkxMGMtNzJiZDd hNTAxZWRkliwidCI6ljJkM2I1MGUwLTZIZjQtNGViYy05MjQ2LTdkMWNiYjc3MDg5YylsImMiOjh $\underline{9}$.

56. Utilización de antihipertensivos en España (1992-2006) [Internet]. Spain: AEMPS; 2009. Available from: https://www.aemps.gob.es/medicamentos-de-uso-humano/observatorio-deuso-de-medicamentos/informes/.

57. Mutharasaiah K, Govindareddy V, Karigar C. Photobiodegradation of halogenated aromatic pollutants. Adv Biosci Biotechnol. 2010; 1: 1-3.

58. Madukasi El, Dai X, He C, Zhou J. Potentials of phototrophic bacteria in treating pharmaceutical wastewater. Int J Environ Sci Technol. 2010; 7: 165-174.

59. Chavan A, Mukherji S. Treatment of hydrocarbon-rich wastewater using oil degrading bacteria and phototrophic microorganisms in rotating biological contactor: Effect of N:P ratio. J Hazard Mater. 2008; 154: 63-72.

60. Hom-Diaz A, Jaén-Gil A, Bello-Laserna I, Rodríguez-Mozaz S, Vicent T, Barceló D, et al. Performance of a microalgal photobioreactor treating toilet wastewater: Pharmaceutically active compound removal and biomass harvesting. Sci Total Environ. 2017; 592: 1-11.

61. Larsen C, Yu ZH, Flick R, Passeport E. Mechanisms of pharmaceutical and personal care product removal in algae-based wastewater treatment systems. Sci Total Environ. 2019; 695: 133772.

62. De Wilt A, Butkovskyi A, Tuantet K, Leal LH, Fernandes TV, Langenhoff A, et al. Micropollutant removal in an algal treatment system fed with source separated wastewater streams. J Hazard Mater. 2016; 304: 84-92.

63. Alkhamis $\mathrm{Y}$, Qin JG. Comparison of $\mathrm{N}$ and $\mathrm{P}$ requirements of Isochrysis galbana under phototrophic and mixotrophic conditions. J Appl Phycol. 2015; 27: 2231-2238.

64. López-Serna R, García D, Bolado S, Jímenez JJ, Lam FY, Golovko O, et al. Photobioreactors based on microalgae-bacteria and purple phototrophic bacteria consortia: A promising technology to reduce the load of veterinary drugs from piggery wastewater. Sci Total Environ. 2019; 692: 259266.

65. Gros M, Cruz-Morato C, Marco-Urrea E, Longree F, Singer H, Sarrà M, et al. Biodegradation of the $X$-ray contrast agent iopromide and the fluoroquinolone antibiotic ofloxacin by the white rot fungus Trametes versicolor in hospital wastewaters and identification of degradation products. Water Res. 2014; 60: 228-241.

66. Lucas D, Castellet-Rovira F, Villagrasa M, Badia-Fabregat M, Barceló D, Vicent $T$, et al. The role of sorption processes in the removal of pharmaceuticals by fungal treatment of wastewater. Sci Total Environ. 2018; 610: 1147-1153.

67. Lucas $D$, Barceló $D$, Rodriguez-Mozaz S. Removal of pharmaceuticals from wastewater by fungal treatment and reduction of hazard quotients. Sci Total Environ. 2016; 571: 909-915. 
68. Wennmalm $\AA$. GB. Public health care management of water pollution with pharmaceuticals: Environmental classification and analysis of pharmaceutical residues in sewage water. Drug Inf J. 2005; 39: 291-297.

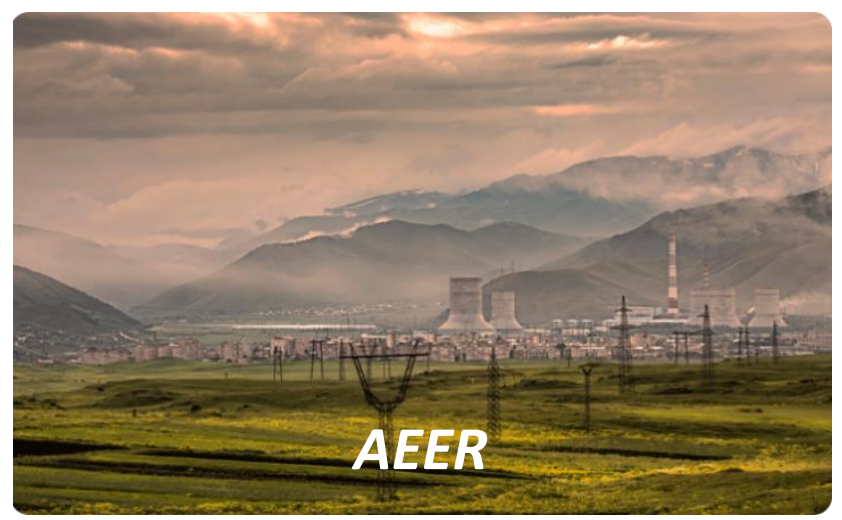

Enjoy AEER by:

1. Submitting a manuscript

2. Joining in volunteer reviewer bank

3. Joining Editorial Board

4. Guest editing a special issue

For more details, please visit:

http://www.lidsen.com/journals/aeer 